\title{
COVID-19 pandemic leads to greater depth of unaffordability of healthy and nutrient-adequate diets in low- and middle-income countries
}

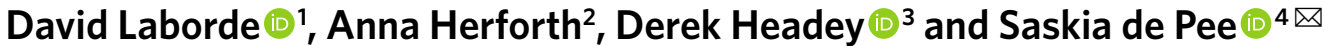

\begin{abstract}
Unaffordability of healthy diets affected 3 billion people before the COVID-19 pandemic, 2.5 billion of whom lived in 63 low- and middle-income countries. In these 63 countries, income losses due to the pandemic have markedly worsened the affordability gap. The proportion of people unable to afford half the cost of a healthy diet increased from $43 \%$ to $50 \%$; this increased unaffordability will aggravate undernutrition, micronutrient deficiencies and diet-related non-communicable diseases.
\end{abstract}

The impact of the COVID-19 pandemic on the economy and people's incomes is severe and has hit people in the informal and service sectors particularly hard, especially in low- and middle-income countries (LMICs). Poverty has increased, and the number of people in acute hunger crisis or worse conditions across 79 countries with World Food Programme operational presence has increased from 130 million to 265 million (ref. ${ }^{1}$ ). Evidence from targeted telephone surveys in Asia and Africa points to disturbingly large increases in poverty and food insecurity throughout $2020^{2,3}$.

Here we assess the further impacts of the COVID-19 pandemic on the problem of not being able to afford healthy and nutrient-adequate diets and on expected changes of food consumption in 63 LMICs using the MIRAGRODEP Computable General Equilibrium model and its extended household-level models. MIRAGRODEP is a global simulation model capturing multiple regions, sectors and international economic linkages, and has recently been used to estimate the impact of pandemic-related economic disruptions at a sectoral, national, regional and global level ${ }^{4}$, and the poverty impacts. The healthy diet is based on food-based dietary guidelines, while the nutrient-adequate diet is based on linear optimization to just meet nutrient needs and, unlike the healthy diet, does not take dietary recommendations, behaviour or food preferences into account. The diet costs and affordability reported by Herforth et al. ${ }^{5}$ were used as a pre-COVID-19 baseline to estimate the change in the number of people who could not afford the healthy and nutrient-adequate diets, and the depth of unaffordability. MIRAGRODEP was then also used to model the pandemic's potential impacts on consumption of different food groups through its effect on income distributions, demand for in-person services and prices.

Of the 3.5 billion people living in the 63 LMICs included in the model, 2.5 billion (70\%) could not afford a healthy diet in a counterfactual 2020 without COVID-19. We estimate that due to the onset of COVID-19 an extra 141 million additional individuals could not afford a healthy diet in 2020 , reaching a total of $74 \%$ of these 63 countries' total population. In 2021 the estimate is an additional 94.6 million (ranging from 21.6 million to 98.6 million for optimistic and pessimistic scenarios, respectively) and in 2022 an additional 32 million (18.4-57.6 million) compared with the no-COVID-19 2020 counterfactual (Fig. 1). The number of people who cannot afford a nutrient-adequate diet, which was 1.3 billion prior to the COVID-19 pandemic (36\% of the countries' population), increased by 220 million (43\%) (data not shown).

Also highly concerning is the greater depth of unaffordability. The proportion of people who could not afford even half the cost of a healthy diet increased from 43\% pre-COVID-19 (2020) to 50\% during COVID-19 (Fig. 2). Similarly, the proportion who could not afford even half the cost of a nutrient-adequate diet increased from $12 \%$ to $17 \%$ (data not shown). For sub-Saharan Africa the unaffordability of a nutrient-adequate diet increased from $61 \%$ to $66 \%$, while the proportion that could not afford even half its cost increased from $28 \%$ to $34 \%$ (data not shown).

Figure 2 shows predicted changes in consumption patterns under the moderate-disruption scenario. Consumption is projected to shift toward increases in vegetable oils, sugar, other crops and other processed foods, while consumption of nutrient-rich foods is projected to decline, along with food away from home (FAFH). These changes primarily reflect households' adaptations to declining income. Fruits, vegetables and animal-source foods are rich in a range of nutrients but are relatively expensive sources of calories ${ }^{6}$. Consumers tend to shift to cheaper sources of calories, including starchy staples, cereals, oils and/or non-perishable processed foods, in the face of income losses, as observed in previous macroeconomic crises $^{7}$ and, conversely, to more nutrient-dense foods when the price of staple food decreases ${ }^{8}$. On the supply-side, COVID19-related disruptions have affected the supply of some perishable foods, especially during lockdowns, and led to rising prices for consumers in some instances ${ }^{3,9}$. Projected increases in the consumption of sugar, oil and processed foods is related to relative prices, but also to stocking-up on non-perishables in anticipation of lockdown measures. Declining consumption of FAFH, which may have a positive or negative effect on health, is also a function of the effects of COVID-19 on reducing demand for in-person services to avoid contagion, as well as lockdowns forcing restaurant closures.

Taken together, these findings suggest that the COVID-19 pandemic has reduced many people's ability to meet their dietary requirements. Not having access to adequate food to meet dietary needs is a key dimension of food insecurity. People who cannot afford healthy diets will necessarily consume lower-quality diets, placing them at risk of malnutrition. Depending on the depth of unaffordability, the prevalence, severity and groups most affected by 
a

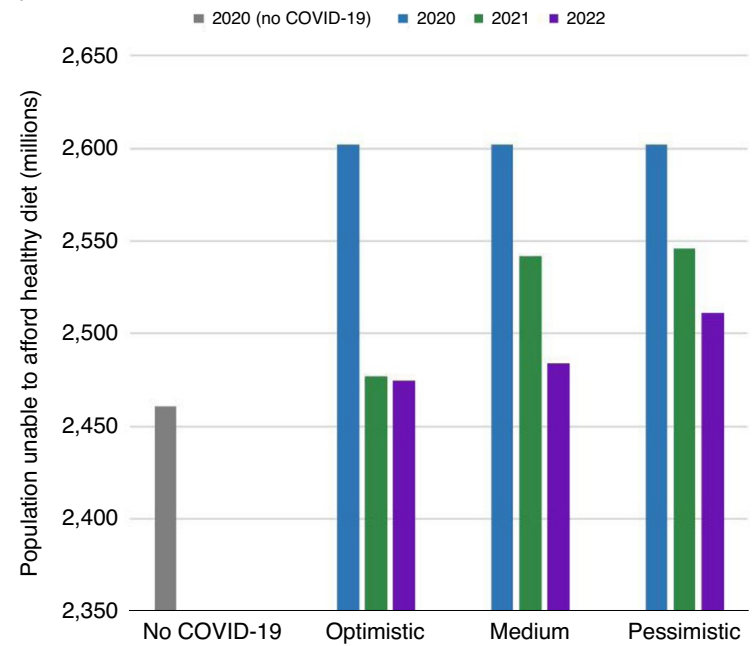

b

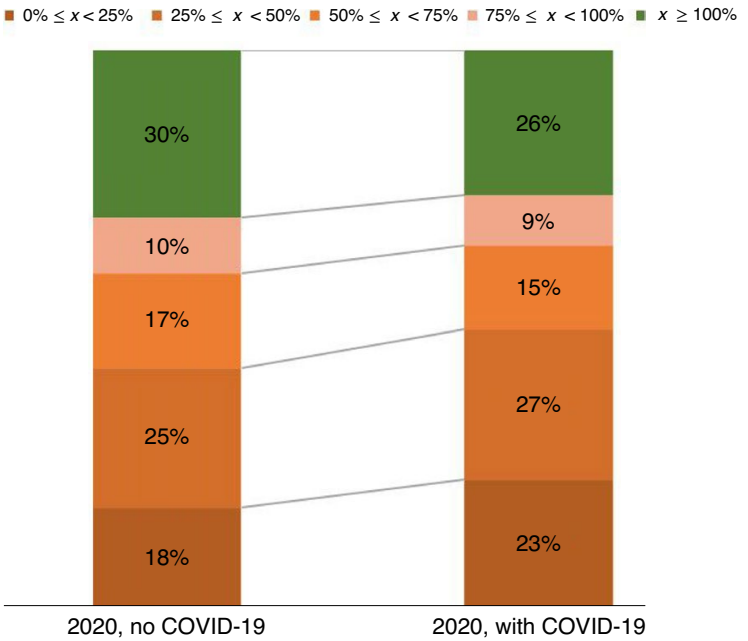

Fig. 1 | Number of people in 63 LMICs who would not be able to afford a healthy diet and the proportion of the cost of a healthy diet that people would be able to afford. a, Number of people (millions) in 2020 (with COVID-19 pandemic), 2021 and 2022, compared with the 2020 no-COVID-19 counterfactual, who would be unable to afford a healthy diet: pessimistic, moderate and optimistic economic disruption scenarios. $\mathbf{b}$, Proportion of people who would be able to afford different proportions of the costs of a healthy diet in the 2020 no-COVID-19 counterfactual and with COVID-19.

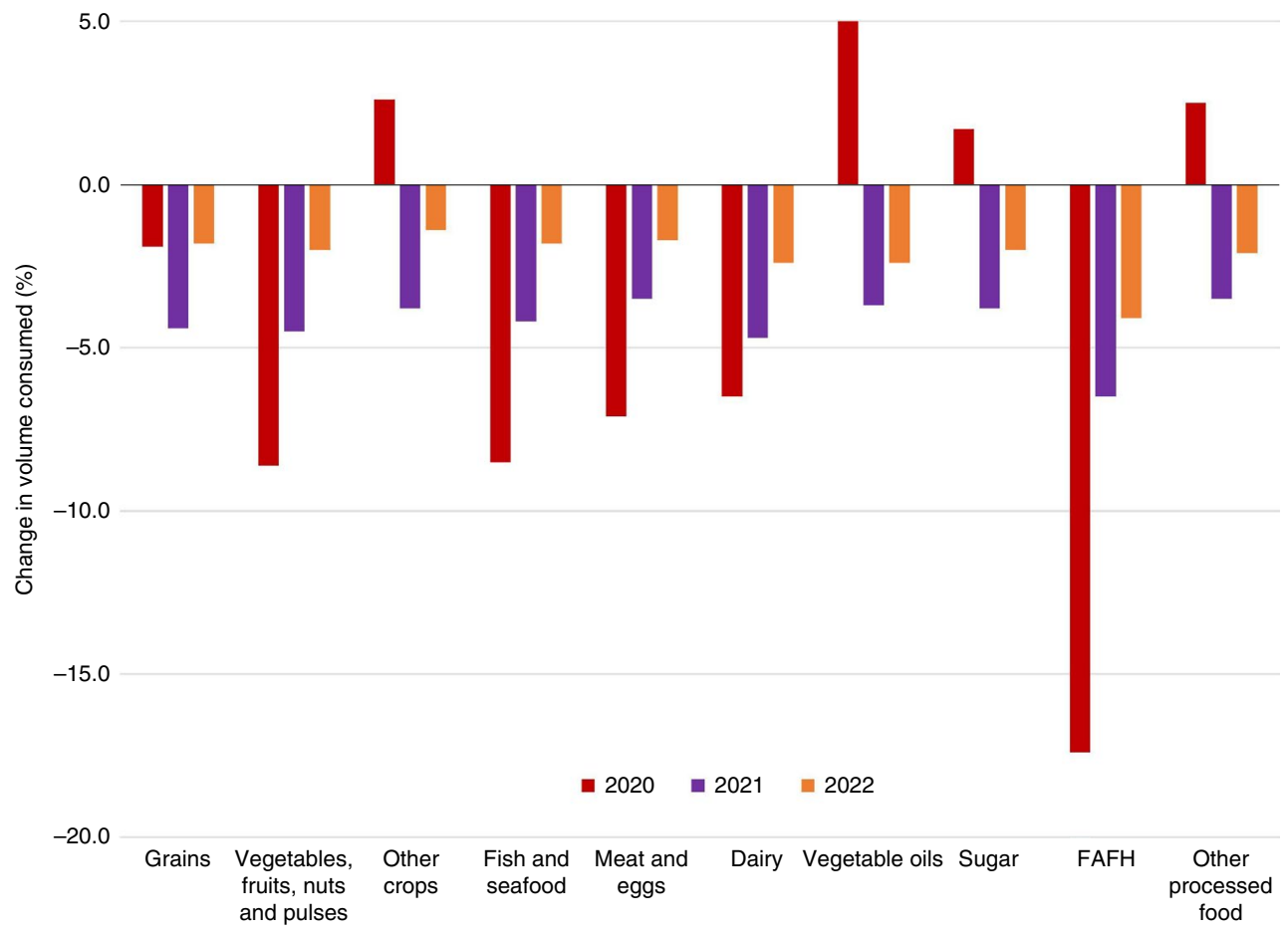

Fig. 2 | Projections of the change in per-capita consumption of various foods in 63 LMICs in 2020, 2021 and 2022 relative to a no-COVID-19 consumption trajectory. 'Grains' includes rice, wheat, maize and other cereals. 'Vegetables, fruits, nuts and pulses' also includes roots and tubers. 'Other crops' also includes cash crops such as coffee, tea and cocoa. 'Other processed food' also includes lightly processed foods (for example, pasta, bread).

different forms of malnutrition vary. Both the depth of unaffordability and the prevailing pattern and risk of different forms of malnutrition should guide a nutrition-focused response to the COVID-19 crisis to prevent the projected increase of malnutrition ${ }^{10}$.

In the short term, efforts to protect household food security through social protection measures are necessary and being implemented ${ }^{11}$. However, the transfers are often quite small ${ }^{12}$ relative to the vast sizes of the populations in need and governments' fiscal space for social protection is constrained by the economic crisis itself. To protect the most nutritionally vulnerable from the consequences of high unaffordability, provision of specific nutritious foods to complement their current dietary intake should be considered $^{12}$. Such specific foods can be distributed at a low price through markets, where feasible, or through social protection and health sector distribution platforms. Beyond the crisis, the cost of healthy diets can be reduced and affordability improved by 
accelerating food system diversification and productivity to increase availability and lower prices of fresh and nutritious foods, as well as through bio- and post-harvest fortification, and inclusive economic growth ${ }^{13}$.

\section{Methods}

To estimate potential effects of the COVID-19 pandemic on household diets, we first estimated changes from 2017 to 2020 in the proportion of the population who would be able to afford a healthy diet. Cost assumptions were derived from an analysis in the 2020 State of Food Security and Nutrition in the World (SOFI) report, which used 2017 food price data for 170 countries to estimate the minimum cost of meeting common diet recommendations, based on food-based dietary guidelines ${ }^{4,13}$. Our analysis included 63 countries for which there are data in MIRAGRODEP and for which the SOFI 2020 report contains information on the cost and affordability of both healthy and nutrient-adequate diets: Albania, Armenia, Bangladesh, Belize, Benin, Bolivia, Botswana, Brazil, Burkina Faso, Burundi, Cape Verde, Colombia, Comoros, Côte d'Ivoire, Djibouti, Ecuador, Egypt, Eswatini, Ethiopia, Ghana, Guinea, Guinea-Bissau, Guyana, Haiti, Honduras, India, Indonesia, Iraq, Kenya, Laos, Lesotho, Liberia, Madagascar, Malawi, Mali, Mauritius, Mongolia, Mozambique, Namibia, Nepal, Nicaragua, Niger, Nigeria, North Macedonia, Panama, Pakistan, Peru, Romania, Rwanda, Senegal, Sierra Leone, South Africa, Sri Lanka, St Lucia, Suriname, Tajikistan, Tanzania, The Gambia, Togo, Uganda, Vietnam, Zambia and Zimbabwe. The MIRAGRODEP model was used to adjust the 2017 SOFI cost estimates to 2020. We assumed that real income distributions and relative food prices did not change between 2017 and 2020, because there had been only modest economic growth during these years, and used this as the no-COVID-19 counterfactual for 2020 . Consistent with a recent report ${ }^{14}$, our assumptions for 2021 and 2022 are that food system disruptions will gradually fade away as sectors continue to adjust, but the macroeconomic and income effects will persist, for which we have modelled an optimistic, moderate and pessimistic scenario. We then applied these three gross national income growth scenarios to estimate the proportion of individuals who could not afford $100 \%, 75 \%, 50 \%$ and $25 \%$ of the cost of a healthy diet and of a nutrient-adequate diet ${ }^{4,13}$, assuming that $63 \%$ of income could be spent on food, following the 2020 SOFI report ${ }^{13}$.

Second, we report MIRAGRODEP projections of per-capita changes in average consumption quantities of 10 aggregated food groups. The predicted shift in food consumption patterns in 2020 due to the COVID-19 pandemic's economic impacts are endogenously determined in the model by three factors: income shocks due to the macroeconomic impacts of COVID-19 translating into food-specific income elasticities; changes in specific COVID-19-related demand behaviours, particularly reduced demand for in-person services such as FAFH; and changes in relative food prices due to logistical and supply chain disruptions during lockdown periods ${ }^{5}$.

\section{Data availability}

General data source. Regarding the data used in the MIRAGRAODEP model, the base-year macroeconomic and sectoral data are based on the GTAP database v.10. The access to this dataset requires a subscription. Base-year poverty numbers are based on the publicly available World Bank Povcalnet http://iresearch. worldbank.org/PovcalNet/povOnDemand.aspx but the full analysis relied on national household surveys, in particular LSMS datasets that require individual authorization (see https://www.worldbank.org/en/programs/lsms for details). Base-year data on diet affordability are based on the SOFI 2020 report $^{5}$.

Information used to build the scenarios. Default macroeconomic projections for the no-COVID scenario are based on the IMF World Economic Outlook from October 2019 publicly available at https://www.imf.org/en/Publications/WEO/ weo-database/2019/October. Data used to build the COVID-19 scenarios on health impacts are based on https://ln2.sync.com/dl/b3fc9ebc0\#36ipcgk35mghvhh4-vi29b9yy-8e688twu and economic disruptions are informed by the Google Community Mobility Reports available at https://www.google.com/ covid19/mobility/.

Core inputs for the 'minimum dataset' that are necessary to interpret, verify and extend the research in the article, transparent to readers. The country- and regional-level macroeconomic and sectoral data based on the MIRAGRODEP model, which provide the inputs to the microeconomic analysis of this paper and are used directly in Fig. 2, for the four scenarios and the three time horizons, are publicly available from a data repository (https://doi.org/10.7910/DVN/MQKA80), together with poverty and diet-affordability national results that support the global results shown in Fig. 1a,b. Source data are provided with this paper.

Received: 9 December 2020; Accepted: 18 June 2021; Published online: 19 July 2021

\section{References}

1. COVID-19 Will Double Number of People Facing Food Crises Unless Swift Action Is Taken (World Food Programme, 2020); https://www.wfp.org/ news/covid-19-will-double-number-people-facing-food-crises-unless-swiftaction-taken

2. Hamadani, J. D. et al. Immediate impact of stay-at-home orders to control COVID-19 transmission on socioeconomic conditions, food insecurity, mental health, and intimate partner violence in Bangladeshi women and their families: an interrupted time series. Lancet Glob. Health $\mathbf{8}$, e1380-e1389 (2020).

3. Kansiime, M. K. et al. COVID-19 implications on household income and food security in Kenya and Uganda: findings from a rapid assessment. World Dev. 137, 105199 (2021).

4. Laborde, D., Martin, W. \& Vos, R. Impacts of COVID-19 on global poverty, food security and diets. Agric. Econ. https://doi.org/10.1111/ agec.12624 (2021).

5. Herforth, A. et al. Cost and Affordability of Healthy Diets Across and Within Countries: Background Paper for The State of Food Security and Nutrition in the World 2020 (Food and Agriculture Organization, 2020); https://doi.org/ $10.4060 / \mathrm{cb} 2431$ en

6. Headey, D. D. \& Alderman, H. H. The relative caloric prices of healthy and unhealthy foods differ systematically across income levels and continents. J. Nutr. 149, 2020-2033 (2019).

7. Block, S. et al. Macro shocks and micro outcomes: child nutrition during Indonesia's crisis. Econ. Hum. Biol. 2, 21-44 (2004).

8. Torlesse, H., Kiess, L. \& Bloem, M. W. Association of household rice expenditure with child nutritional status indicates a role for macroeconomic food policy in combating malnutrition. J. Nutr. 133, 1320-1325 (2003).

9. Laborde, D. \& Smaller. C. What Would it Cost to Avert the COVID-19 Hunger Crisis? (Cornell Univ. Library, 2020); https://ecommons.cornell.edu/ handle/1813/70172

10. Osendarp, S. et al. The COVID-19 crisis will exacerbate maternal and child undernutrition and child mortality in low- and middle-income countries. Nat. Food https://doi.org/10.1038/s43016-021-00319-4 (2021).

11. Supporting National Social Protection Responses to the Socioeconomic Impact of COVID-19 (World Food Programme, 2020); https://www.wfp.org/publications/ supporting-national-social-protection-responses-socioeconomic-impactcovid-19

12. Maximizing Social Protection's Contribution to Human Capital Development. Fill the Nutrient Gap Analysis (World Food Programme, 2020); https://docs. wfp.org/api/documents/WFP-0000113930/download/?.ga= 2.205917395.1168755595.1607265237-633098164.1591685439

13. FAO, IFAD, UNICEF, WFP, WHO. The State of Food Security and Nutrition in the World 2020. Transforming Food Systems for Affordable Healthy Diets (Food and Agriculture Organization, 2020).

14. Laborde, D., Parent, M. \& Smaller, C. Ending Hunger, Increasing Incomes, and Protecting the Climate: What Would it Cost Donors? (Cornell Univ. Library, 2020); https://ecommons.cornell.edu/handle/1813/72864

\section{Acknowledgements}

We thank the Standing Together for Nutrition consortium for inputs provided during the preparation of this paper.

\section{Author contributions}

S.d.P. and A.H. conceptualized the analytical question. D.H. and D.L. designed the analysis. D.L. conducted the analysis. All authors interpreted the data. S.d.P. drafted the paper, and all authors reviewed and approved the submitted version and agreed to be personally accountable for their own contributions.

\section{Competing interests}

The authors declare no competing interests.

\section{Additional information}

Supplementary information The online version contains supplementary material available at https://doi.org/10.1038/s43016-021-00323-8.

Correspondence and requests for materials should be addressed to S.d.P.

Peer review information Nature Food thanks Shenggen Fan and Amelia Finaret for their contribution to the peer review of this work.

Reprints and permissions information is available at www.nature.com/reprints.

Publisher's note Springer Nature remains neutral with regard to jurisdictional claims in published maps and institutional affiliations.

(C) The Author(s), under exclusive licence to Springer Nature Limited 2021 